\title{
Smith-Purcell radiation emission in aligned nanoparticles
}

\author{
F. J. García de Abajo* \\ Materials Sciences Division, Lawrence Berkeley National Laboratory, Berkeley, California 94720
}

(Received 19 August 1999)

\begin{abstract}
Smith-Purcell (SP) radiation produced by interaction of fast electron beams running parallel to strings of nanoparticles is investigated. Results for the radiation emission probability and electron energy loss spectra using finite and infinite strings of $\mathrm{Al}$ and silica spheres are presented. Both of these quantities are obtained by solving Maxwell's equations exactly using a multipole expansion approach. The response of the spheres is described in terms of their local frequency-dependent dielectric functions. In silica, the emission probability is seen to coincide with the energy loss probability within the gap region, where the solid cannot absorb any energy. Large emission rates are predicted for $\mathrm{Al}$, suggesting its possible application in tunable soft uv light generation. The dependence of the emission on the size of the spheres, the string period, and the electron energy is discussed in detail. Finite size effects are also studied for strings of 1-15 Al spheres.
\end{abstract}

PACS number(s): 41.60.- m, 42.25.Fx, 61.16.Bg

\section{INTRODUCTION}

When an electron moves parallel to an optical grating, it is capable of emitting radiation along directions that depend on the emitted photon energy. This is the well-known SmithPurcell (SP) effect [1-16], first reported nearly half a century ago [1]. Successive experimental demonstrations of the effect were carried out since then using 30-300 keV electrons to produce light of wavelength in the $\mu \mathrm{m}$ region [2,3]. Relativistic electrons have been also employed to generate $\mathrm{mm}$ radiation $[8,13]$, very intense along the forward direction [11]. Recently, 20-40 keV electron beams produced by conventional electron microscopes and coupled to metallic gratings of periods in the submillimeter range have been proved to be an efficient tool for generating far infrared radiation $[14,15]$.

Early theoretical descriptions of the SP effect relied on the diffraction of the evanescent waves associated with the moving electron $[17,4,5,10]$, and more recently, it has been emphasized that an equivalent description results from the currents induced on the grating surface by the passage of the electron [12]. Since these induced currents have to follow the curved profile of the grating, they are modulated in time, giving rise to the emission of radiation. Theoretical studies of the SP effect have been devised with a view to be applied to produce X-rays [9] and also in the acceleration of electrons [6]. Most of the theoretical treatments of the SP effect assume that the grating can be considered to be perfectly conducting, though some effort has been made to include plasma oscillations in the response of the grating when the energy of the emitted radiation matches the plasma frequency [7].

The present work is intended to incorporate the effect of the frequency dependence of the response function of the material that couples to the electron to produce SP radiation. This plays a central role in the examples that follow, where radiation of $1-15 \mathrm{eV}$ is examined, for which the behavior

\footnotetext{
*Permanent address: Departamento de CCIA, Facultad de Informática, Donostia International Physics Center, and Centro Mixto CSIC-UPV/EHU, San Sebastián, Spain. Electronic address: ccpgaabf@photon.lbl.gov
}

of the materials used here is far from being that of a perfect metal. Local frequency-dependent dielectric functions constitute an excellent approximation when the electrons travel outside the materials under consideration, in which case the effect of dispersion in the response function is not important [18].

The energy loss suffered by the electrons has been investigated as well in order to determine the fraction that is transformed into radiation. Following previous discussions of electron energy loss spectra [19-23], the loss probability has been obtained from the retarding force produced by the induced field acting back on the electron. In this context, relativistic results based upon numerical simulation have been reported for the energy loss and the radiation emission probabilities due to the interaction of fast electrons with planes of spheres $[24,25]$. A nonrelativistic analytical treatment of the energy loss spectra in the presence of an infinite set of aligned spheres has also been given recently [26].

The geometry considered here consists of periodic strings of identical finite objects, for which an analytical relativistic description is offered. Advances towards a practical realization of this geometry are being produced in the context of quantum dots on the nanometer scale [27]. The method used to calculate radiation emission probabilities consists in solving exactly the Maxwell equations by using a multiple scattering technique [28] that permits us to express the response of a collection of dielectric objects in terms of their individual scattering properties. Although this method can be applied to arbitrary objects, strings of spheres are considered in this work for simplicity, since the scattering matrices become analytical in that case [29,30].

The details of the theory of SP radiation produced by the interaction of fast electrons with aligned strings of dielectric finite objects are presented in Sec. II. Numerical results for strings formed by spheres of $\mathrm{Al}$ and $\mathrm{SiO}_{2}$ are offered in $\mathrm{Sec}$. III. Finally, the main conclusions are summarized in Sec. IV. Gaussian atomic units (a.u., that is, $e=m=\hbar=1$ ) will be used from now on, unless otherwise specified.

\section{THEORY}

A theory is presented in this section to calculate electron energy loss and photon emission probabilities for the geometry illustrated in Fig. 1. An electron is assumed to be mov- 


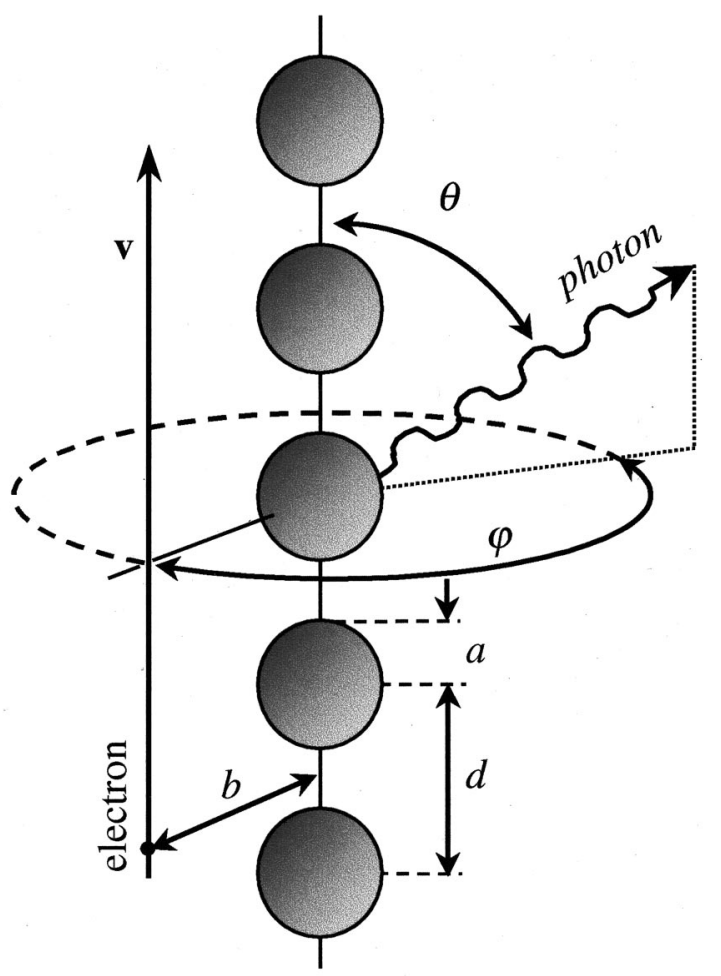

FIG. 1. Schematic representation of the geometry considered in this work. An electron is traveling parallel to an infinite periodic string of dielectric objects with velocity $\mathbf{v}$ and impact parameter $b$ with respect to the axis of symmetry of the string, chosen to be the $z$ axis. As a result of the interaction between the string and the electron, radiation is emitted along directions $(\theta, \varphi)$ with frequency $\omega$ such that the phase difference between the contributions to the far field coming from contiguous objects is a multiple of $2 \pi$ [i.e., $\omega d(1 / v-\cos \theta / c)=-2 \pi n$, where $d$ is the string spacing]. The present formalism can be applied to strings of arbitrary objects, provided one knows their scattering matrix $t$. For simplicity, the particular case of spheres of radius $a$ has been considered here.

ing parallel to an infinite periodic string of dielectric objects. The interaction between the fast electron and the string produces induced charges and currents in the objects, and as a result, radiation is emitted over a continuum spectrum.

Expressions for the photon emission and electron energy loss probabilities will be obtained in Secs. II C and II D, respectively, employing a general multiple scattering technique to calculate the induced electromagnetic field. This technique is reviewed in Sec. II A for an arbitrary cluster $[28,31]$. The application to the particular case of a periodic string is presented in Sec. II B.

\section{A. Multiple scattering approach to the electromagnetic problem}

The method employed here to find the electromagnetic field in the presence of a cluster of dielectric objects is based upon multiple elastic scattering of multipole expansions (MESME) around the positions of the objects of the cluster $[28,31]$. The electric and magnetic fields in the vacuum region surrounding the cluster can be written in frequency space $\omega$ in terms of magnetic and electric scalar functions $\psi_{\alpha}^{M}$ and $\psi_{\alpha}^{E}$ as $[32,30]$

$$
\mathbf{E}=\mathbf{L}_{\alpha} \psi_{\alpha}^{M}-\frac{i}{k} \nabla \times \mathbf{L}_{\alpha} \psi_{\alpha}^{E}
$$

and

$$
\mathbf{H}=-\frac{i}{k} \boldsymbol{\nabla} \times \mathbf{L}_{\alpha} \psi_{\alpha}^{M}-\mathbf{L}_{\alpha} \psi_{\alpha}^{E},
$$

where $k=\omega / c$ and $\mathbf{L}_{\alpha}=-i\left(\mathbf{r}-\mathbf{r}_{\alpha}\right) \times \nabla$ is the orbital angular momentum operator relative to a given position $\mathbf{r}_{\alpha}$. Similar to the electromagnetic field, the scalar functions satisfy the wave equation $\left(\nabla^{2}+k^{2}\right) \psi=0$, and therefore, they have to be made of free plane waves. In particular, for the the external field, they can be expanded in terms of spherical harmonics $Y_{L}$ as

$$
\psi_{\alpha}^{\mathrm{ext}}(\mathbf{r})=\sum_{L} j_{L}\left[k\left(\mathbf{r}-\mathbf{r}_{\alpha}\right)\right] \psi_{\alpha, L}^{\mathrm{ext}},
$$

where $L=(l, m), j_{L}(\mathbf{u})=i^{l} j_{l}(|\mathbf{u}|) Y_{L}(\hat{\mathbf{u}}), \quad j_{l}$ is a spherical Bessel function, and $\psi_{\alpha}$ groups both magnetic and electric components. Equation (3) is a consequence of the assumption that no external sources are present in the vacuum region under consideration, so that the external field cannot lead to a net energy flux through any closed surface.

The objects of the cluster will be labeled by nearby positions $\mathbf{r}_{\alpha}$. Single scattering (SS) of the external field on one of those objects (e.g., $\alpha$ ) produces induced charges and currents that set up a scattered field whose corresponding scalar functions can be written as [28]

$$
\psi_{\alpha}^{\mathrm{SS}}(\mathbf{r})=\sum_{L} h_{L}^{(+)}\left[k\left(\mathbf{r}-\mathbf{r}_{\alpha}\right)\right] \psi_{\alpha, L}^{\mathrm{SS}},
$$

where $h_{L}^{(+)}(\mathbf{u})=i^{l} h_{l}^{(+)}(|\mathbf{u}|) Y_{L}(\hat{\mathbf{u}})$ and $h_{l}^{(+)}$is a Hankel function [33]. This equation is valid for a sphere centered at $\mathbf{r}_{\alpha}$ and fully containing object $\alpha$ (i.e., containing the induced charges and currents).

In the linear response approximation, the SS field is proportional to the external field, so that

$$
\psi_{\alpha, L}^{\mathrm{SS}}=\sum_{L^{\prime}} t_{\alpha, L L^{\prime}} \psi_{\alpha, L}^{\mathrm{ext}},
$$

where $t_{\alpha, L L^{\prime}}$ is the so-called scattering matrix. Explicit expressions for $t_{\alpha, L L^{\prime}}$ can be found in the case of both homogeneous spheres and coated spheres [29,30], for which $t_{\alpha, L L^{\prime}}=t_{\alpha, l} \delta_{L L^{\prime}}$ and magnetic and electric components are decoupled (see Sec. III).

The field induced by the presence of the cluster can be regarded as the result of self-consistent scattering of the external field, and therefore, it can be expanded into multipoles in the same way as the SS field in Eq. (4). The total induced field is given by the sum

$$
\psi^{\text {ind }}(\mathbf{r})=\sum_{\alpha, L} h_{L}^{(+)}\left[k\left(\mathbf{r}-\mathbf{r}_{\alpha}\right)\right] \psi_{\alpha, L}^{\text {ind }},
$$

where $\alpha$ runs over cluster objects. Moreover, the field induced by each object $\alpha$ is the sum of the SS contribution plus the result of the propagation of the field induced by every other object $\beta \neq \alpha$ from $\beta$ to $\alpha$, followed by scattering 
at $\alpha$. This leads to the self-consistent relation [28]

$$
\widetilde{\psi}_{\alpha}^{\text {ind }}=\widetilde{\psi}_{\alpha}^{\text {SS }}+t_{\alpha} \sum_{\beta \neq \alpha} H_{\alpha \beta} \widetilde{\psi}_{\beta}^{\text {ind }},
$$

where matrix notation has been adopted (i.e., $\widetilde{\psi}_{\alpha}^{\text {SS(ind) }}$ is the vector of components $\psi_{\alpha, L}^{\text {SS (ind) }}$, and $t_{\alpha}$ is the matrix of components $\left.t_{\alpha, L L^{\prime}}\right)$ and the operator $H_{\alpha \beta}$ accounts for the noted propagation. The latter can be written as $[28,31]$

$$
H_{\alpha \beta}=R_{\alpha \beta}^{-1} T_{\alpha \beta}^{z} G_{\alpha \beta}^{z} R_{\alpha \beta},
$$

where $R_{\alpha \beta}$ is a rotation matrix [33] that acts on the spherical harmonics of the multipole expansions and is defined such that it brings the bond vector $\mathbf{r}_{\alpha}-\mathbf{r}_{\beta}$ onto the positive $z$ axis; the matrix $G_{\alpha \beta}^{z}$ of components

$$
\begin{aligned}
G_{\alpha \beta, L L^{\prime}}^{z}= & \delta_{m m^{\prime}} \sqrt{4 \pi} \sum_{l^{\prime \prime}=\left|l-l^{\prime}\right|}^{l+l^{\prime}} \sqrt{2 l^{\prime \prime}+1} \\
& \times i^{l^{\prime \prime}} h_{l^{\prime \prime}}^{(+)}\left(k\left|\mathbf{r}_{\alpha}-\mathbf{r}_{\beta}\right|\right)\left\langle l^{\prime} m\left|l^{\prime \prime} 0\right| l m\right\rangle
\end{aligned}
$$

propagates spherical harmonics from $\beta$ to $\alpha$ [34];

$$
\left\langle L^{\prime}\left|L^{\prime \prime}\right| L\right\rangle=\int d \Omega Y_{L^{\prime}}^{*}(\Omega) Y_{L^{\prime \prime}}(\Omega) Y_{L}(\Omega)
$$

is a Gaunt integral; and the following operator is defined to compensate for the lack of invariance of multipoles under translations of their origin [28,31]:

$$
T_{\alpha \beta, L L^{\prime}}^{z}=\left[\begin{array}{ll}
\delta_{L L^{\prime}} & 0 \\
0 & \delta_{L L^{\prime}}
\end{array}\right]+\delta_{m m^{\prime}} k\left|\mathbf{r}_{\alpha}-\mathbf{r}_{\beta}\right|\left[\begin{array}{cc}
p_{l l^{\prime} m} & q_{l l^{\prime} m} \\
-q_{l l^{\prime} m} & p_{l l^{\prime} m}
\end{array}\right],
$$

where

$$
\begin{gathered}
q_{l l^{\prime} m}=\frac{m \delta_{l, l^{\prime}}}{l(l+1)}, \\
p_{l l^{\prime} m}=\delta_{l+1, l^{\prime}} D_{l+1, m}-\delta_{l-1, l^{\prime}} D_{L},
\end{gathered}
$$

and

$$
D_{L}=\frac{i}{l} \sqrt{\frac{(l+m)(l-m)}{(2 l-1)(2 l+1)}} .
$$

Notice that $G_{\alpha \beta}^{z}$ does not mix electric and magnetic components, whereas $T_{\alpha \beta}^{z}$ acts on the two-component scalar functions

$$
\left[\begin{array}{l}
\psi^{M} \\
\psi^{E}
\end{array}\right]
$$

mixing electric and magnetic parts via off-diagonal terms $q_{l l^{\prime} m}$.

\section{B. Application to an infinite string}

Let us consider a periodic string of identical objects centered around positions $\mathbf{r}_{\alpha}=(0,0, \alpha d)$, where $d$ is the spacing and $\alpha$ runs over the integral numbers (see Fig. 1). An external electron will be taken to be moving along the trajectory described by $\mathbf{r}_{t}=(b, 0, v t)$, where $b$ is the impact parameter with respect to the axis of the string.

Equation (3) is valid inside the region free of external sources defined by the sphere of radius $b$ centered around $\mathbf{r}_{\alpha}$. More precisely, the coefficients $\psi_{\alpha, L}^{\text {ext }}$ are given by $[30,31]$

$$
\psi_{\alpha, L}^{\mathrm{ext}}=\phi_{L}^{\mathrm{ext}} e^{i \alpha \omega d / v}
$$

where

$$
\left[\begin{array}{c}
\phi_{L}^{M, \text { ext }} \\
\phi_{L}^{E \text {,ext }}
\end{array}\right]=\frac{-2 \pi i^{1-l} k}{l(l+1) c} K_{m}\left[\frac{\omega b}{v \gamma}\right]\left[\begin{array}{c}
2 m A_{L}^{+} v / c \\
B_{L} / \gamma
\end{array}\right],
$$

$K_{m}$ is the modified Bessel function of order $m, \gamma$ $=1 / \sqrt{1-(v / c)^{2}}$, and $A_{L}^{+}$and $B_{L}$ are coefficients that depend exclusively on the ratio $v / c$. In particular, $A_{L}^{+}$is implicitly defined by

$$
\int d t e^{i \omega t} h_{L}^{(+)}[k(b, 0, v t) \mid]=i^{l} \frac{A_{L}^{+}}{\omega} K_{m}\left(\frac{\omega b}{v \gamma}\right) .
$$

Explicit expressions for $A_{L}^{+}$and $B_{L}$ have been given elsewhere [30,31] and they can be constructed in terms of Gegenbauer polynomials $C_{\nu}^{\mu}$ as $[35,36]$

$$
\begin{aligned}
A_{L}^{+}= & i^{l+m}(2 m-1) ! ! \sqrt{\frac{(2 l+1)}{\pi} \frac{(l-m) !}{(l+m) !}} \\
& \times \frac{(c / v)^{m+1}}{\gamma^{m}} C_{l-m}^{m+1 / 2}(c / v), \quad m \geqslant 0,
\end{aligned}
$$

making use of $A_{l,-m}^{+}=(-1)^{m} A_{L}^{+}$for $m<0$, and

$$
\begin{aligned}
B_{L}= & A_{l, m+1}^{+} \sqrt{(l+m+1)(l-m)} \\
& -A_{l, m-1}^{+} \sqrt{(l-m+1)(l+m)} .
\end{aligned}
$$

The periodicity of the string is reflected in the fact that $H_{\alpha \beta}$ depends on $\alpha$ and $\beta$ only through the difference $\alpha$ $-\beta$. Actually, $\left|\mathbf{r}_{\alpha}-\mathbf{r}_{\beta}\right|=d|\alpha-\beta|$ in Eqs. (8) and (9). In addition, the rotation matrices reduce to [33]

$$
R_{\alpha \beta, L L^{\prime}}=\left\{\begin{array}{l}
\delta_{L L^{\prime}}, \quad \alpha>\beta \\
(-1)^{l+m} \delta_{l l^{\prime}} \delta_{m,-m^{\prime}}, \quad \alpha<\beta .
\end{array}\right.
$$

Therefore, using Eq. (7), one has that $H_{\alpha \beta}$ depends on $\alpha$ and $\beta$ only through $\alpha-\beta$. This, together with Eq. (10), permits us to recast Eq. (6) as

$$
\widetilde{\phi}^{\text {ind }}=\frac{1}{1-t H(\omega / v)} t \widetilde{\phi}^{\text {ext }},
$$

where the $\alpha$ dependence has been dropped from $t$ since all objects of the string are assumed to be identical,

$$
H(q)=\sum_{\alpha \neq 0} H_{\alpha 0} e^{-i \alpha q d},
$$


and $\widetilde{\phi}^{\text {ind }}$ is implicitly defined by the coefficients of the multipole expansion of $\psi^{\text {ind }}$ [see Eq. (5)],

$$
\psi_{\alpha, L}^{\text {ind }}=\phi_{L}^{\text {ind }} e^{i \alpha \omega d / v} .
$$

Equation (15) is a consequence of the periodicity of the system and the assumption that the electron is moving with constant velocity parallel to the string. Notice that the dependence of $\psi_{\alpha}^{\text {ind }}$ and $\psi_{\alpha}^{\text {ext }}$ on the object $\alpha$ comes exclusively via the phase factors $\exp (i \alpha \omega d / v)$.

The operator $H$ in Eq. (13) depends on both the string spacing and the electron velocity. However, it is independent of the particular shape and composition of the objects that made up the string. This latter information is fully contained inside the scattering matrix $t$.

Using Eqs. (7), (8), (9), (12), and (14), the components of $H$ are found to be

$$
H_{L L^{\prime}}(q)=\delta_{m m^{\prime}} H_{l l^{\prime}}^{m}(q),
$$

where

$$
\begin{gathered}
H_{l l^{\prime}}^{m}(q)=4 \pi \sum_{l^{\prime \prime}=\left|l-l^{\prime}\right|}^{l+l^{\prime}} \sqrt{2 l^{\prime \prime}+1}\left[\begin{array}{cc}
f_{l l^{\prime} l^{\prime \prime}}^{m} & g_{l l^{\prime} l^{\prime \prime}}^{m} \\
-g_{l l^{\prime} l^{\prime \prime}}^{m} & f_{l l^{\prime} l^{\prime \prime}}^{m}
\end{array}\right], \\
f_{l l^{\prime} l^{\prime \prime}}^{m}=\left\langle L\left|l^{\prime \prime} 0\right| l^{\prime} m\right\rangle\left(\eta_{l^{\prime \prime}}^{-}+\eta_{l^{\prime \prime}}^{+}\right)+\left[D_{l+1, m}\left\langle l+1, m\left|l^{\prime \prime} 0\right| l^{\prime} m\right\rangle\right. \\
\left.-D_{L}\left\langle l-1, m\left|l^{\prime \prime} 0\right| l^{\prime} m\right\rangle\right]\left(\zeta_{l^{\prime \prime}}^{-}+\zeta_{l^{\prime \prime}}^{+}\right), \\
g_{l l^{\prime} l^{\prime \prime}}^{m}=\left\langle L\left|l^{\prime \prime} 0\right| l^{\prime} m\right\rangle \frac{m}{l(l+1)}\left(\zeta_{l^{\prime \prime}}^{-}-\zeta_{l^{\prime \prime}}^{+}\right),
\end{gathered}
$$

and

$$
\left[\begin{array}{c}
\eta_{l}^{ \pm} \\
\zeta_{l}^{ \pm}
\end{array}\right]=\sum_{\alpha=1}^{\infty} i^{l} h_{l}^{(+)}(\alpha k d) e^{ \pm i \alpha q d}\left[\begin{array}{c}
1 \\
\alpha k d
\end{array}\right] .
$$

Equation (16) involves slowly convergent series (i.e., the general term goes as $1 / \alpha$ in the upper part and 1 in the lower part times phase factors for large values of $\alpha$ ). However, the functions $\eta_{l}^{ \pm}$and $\zeta_{l}^{ \pm}$can be efficiently calculated using the identities

$$
\begin{aligned}
\eta_{l}^{ \pm}= & -\frac{1}{2 k d}\left\{\ln \left[2\left(1-\cos \varpi^{ \pm}\right)\right]+i\left(\varpi^{ \pm}-\pi\right)\right\} \\
& +\sum_{\alpha=1}^{\infty}\left[i^{l} h_{l}^{(+)}(\alpha k d)-\frac{e^{i \alpha k d}}{\alpha k d}\right] e^{ \pm i \alpha q d}
\end{aligned}
$$

and

$$
\begin{aligned}
\zeta_{l}^{ \pm}= & \frac{1}{e^{-i \varpi^{ \pm}}-1}-\frac{i l(l+1)}{4 k d}\left\{\ln \left[2\left(1-\cos \varpi^{ \pm}\right)\right]\right. \\
& \left.+i\left(\varpi^{ \pm}-\pi\right)\right\}+\sum_{\alpha=1}^{\infty}\left\{i^{l} \alpha k d h_{l}^{(+)}(\alpha k d)\right. \\
& \left.-\left[1+\frac{i l(l+1)}{2 \alpha k d}\right] e^{i \alpha k d}\right\} e^{ \pm i \alpha q d},
\end{aligned}
$$

where $\varpi^{ \pm}=[(k \pm q) d] \bmod 2 \pi$ and an infinitesimally-small positive imaginary part has been added to $k$. This is consistent with the retarded response formalism implicitly assumed in Eqs. (4), (5), and (8) (i.e., the choice of outgoing waves $h_{L}^{(+)}$rather than incoming waves). The sums in Eqs. (17) and (18) converge faster than that in Eq. (16), since their terms die off as $1 / \alpha^{2}$ when $\alpha \rightarrow \infty$.

As expected, when the sphere separation is very large ( $k d \gg 1$ ), the sum in Eq. (16) vanishes and $H \simeq 1$. Then, the isolated sphere limit is recovered from Eq. (13).

\section{Radiation emission}

Inserting the multipole expansion of the induced field into Eqs. (1) and (2), and making use of Eqs. (5) and (15), the far electric and magnetic fields reduce to $[37,31]$

$$
\mathbf{E}^{\text {ind }} \simeq \mathbf{f}(\Omega) \frac{e^{i k r}}{r}, \quad r \rightarrow \infty
$$

and

$$
\mathbf{H}^{\text {ind }} \simeq \hat{\mathbf{r}} \times \mathbf{f}(\Omega) \frac{e^{i k r}}{r}, \quad r \rightarrow \infty,
$$

where $\mathbf{f}$ is normal to $\mathbf{r}$, since these are radiative fields that decay with the distance as $1 / r$. In the case under consideration, one finds

$$
\mathbf{f}(\Omega)=\chi(\Omega) \sum_{\alpha} \frac{e^{i \alpha \Delta}}{k},
$$

where

$$
\chi(\Omega)=\sum_{L}\left[\phi_{L}^{M, \text { ind }} \mathbf{L} Y_{L}(\Omega)+\phi_{L}^{E \text {,ind }} \hat{\mathbf{r}} \times \mathbf{L} Y_{L}(\Omega)\right],
$$

$$
\begin{aligned}
\mathbf{L} Y_{L}(\Omega)= & {\left[\frac{C_{+}}{2} Y_{l m+1}(\Omega)+\frac{C_{-}}{2} Y_{l m-1}(\Omega),\right.} \\
& \left.-\frac{i C_{+}}{2} Y_{l m+1}(\Omega)+\frac{i C_{-}}{2} Y_{l m-1}(\Omega), m Y_{L}(\Omega)\right]
\end{aligned}
$$

are vector spherical harmonics

$$
\Delta=\omega d\left(1 / v-\frac{\cos \theta}{c}\right),
$$

and $\Omega=(\theta, \varphi)$ denotes the polar angles of $\mathbf{r}$ with respect to the electron trajectory (see Fig. 1).

The probability of emitting a photon due to the interaction of the string with the external electron can be obtained by integrating the Poynting vector over a sphere of arbitrarilylarge radius $r$. One finds that the probability of emission per unit of energy range and unit of solid angle around the direction $\Omega$ is given by $[37,30]$ 


$$
\begin{aligned}
\Gamma^{\mathrm{rad}}(\omega, \Omega) & =\frac{r^{2}}{4 \pi^{2} k} \operatorname{Re}\left\{\left[\mathbf{E}^{\text {ind }}(\omega) \times \mathbf{H}^{\text {ind }}(-\omega)\right] \cdot \hat{\mathbf{r}}\right\} \\
& =\frac{1}{4 \pi^{2} k}|\mathbf{f}(\Omega)|^{2} .
\end{aligned}
$$

The summation over $\alpha$ coming from $\mathbf{f}$ in Eq. (23) can be performed using the identity

$$
\begin{aligned}
\left|\sum_{\alpha=1}^{N} e^{i \alpha \Delta}\right|^{2} & =\frac{\sin ^{2}(N \Delta / 2)}{\sin ^{2}(\Delta / 2)} \\
& \simeq 2 \pi N \sum_{n} \delta(\Delta+2 \pi n), \quad N \rightarrow \infty,
\end{aligned}
$$

where the sum on the right hand side runs over integers $n$. Noticing that $n$ can only take negative values since $\Delta>0$ [see Eq. (22)], one finds, upon integration over $\theta$, that the probability of emitting a photon per sphere and unit of azimuthal angle $\varphi$ reduces to

$$
\frac{\Gamma^{\mathrm{rad}}(\omega, \varphi)}{N}=\frac{1}{2 \pi k^{4} d} \sum_{n=-\infty}^{-1}\left|\chi\left(\Omega_{n}\right)\right|^{2} .
$$

For each harmonic $n$, the emission takes place along a cone whose aperture depends on the photon frequency $\omega$ and is given by the polar directions $\Omega_{n}=\left(\theta_{n}, \varphi\right)$, where [1]

$$
\theta_{n}=\cos ^{-1}\left[\frac{c}{v}+\frac{2 \pi n}{\omega d / c}\right]
$$

Equation (26) responds to the intuitive argument that the emission can only take place along those directions for which the far field originating in the objects of the string is constructive, and therefore, the difference in phase between contiguous objects, given by Eq. (22), has to be a multiple of $2 \pi$.

A threshold energy is obtained for each $n$ when $\theta=\pi$ (backward emission), and an absolute threshold is found for $n=-1$, given by $\omega_{0}=(2 \pi c / d) /(c / v+1)$. This can be clearly observed in the emission spectra represented in Figs. 2-6. In particular, Fig. 5(a) shows the dependence of the polar angle of emission on photon energy for $n=-1$. Notice that the energy of emission for a given angle $\theta$ is proportional to $|n|$ and inversely proportional to $d$. Therefore, when $d \rightarrow \infty$ (isolated sphere limit), the emission can occur for all angles and energies, and high values of $|n|$ become relevant.

\section{Electron energy loss}

The energy loss suffered by the electron can be obtained from the induced electric field acting back on it [19-23]. Following previous analyses, one finds $[23,30]$

$$
\Delta E^{\text {loss }}=\int d t \mathbf{v} \cdot \mathbf{E}^{\text {ind }}\left(\mathbf{r}_{t}, t\right)=\int_{0}^{\infty} \omega d \omega \Gamma^{\text {loss }}(\omega),
$$

where

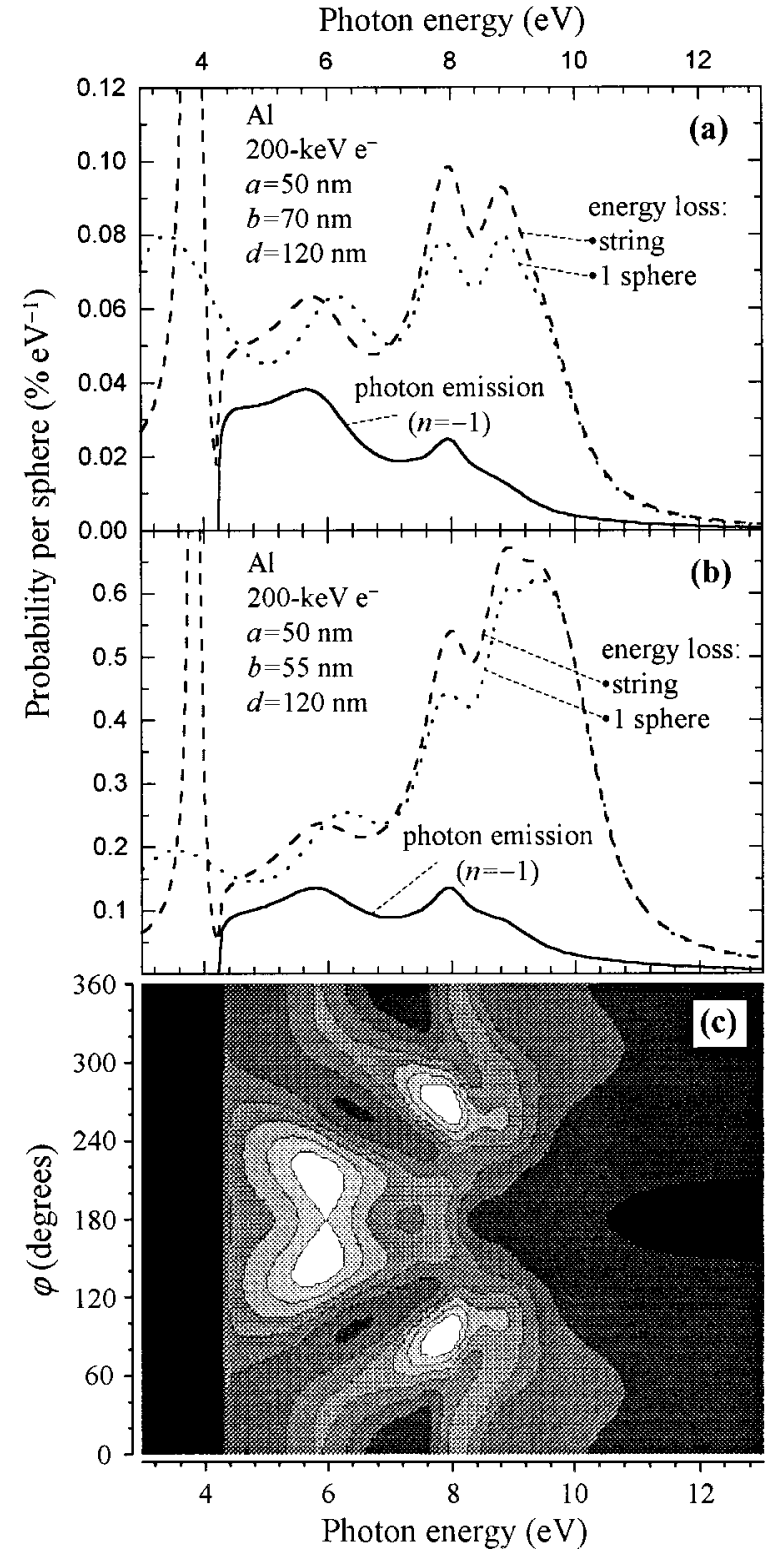

FIG. 2. (a) Energy loss probability and SP radiation emission probability for a $200-\mathrm{keV}$ electron moving parallel to an infinite periodic string of aligned $\mathrm{Al}$ spheres (see Fig. 1). The radius of the spheres is $a=50 \mathrm{~nm}$, the period along the string is $d=120 \mathrm{~nm}$, and the distance from the electron trajectory to the sphere centers is $b=70 \mathrm{~nm}$. The photon emission (energy loss) probability per sphere and energy range is represented by a solid (dashed) curve for the first harmonic $(n=-1$; see text). The energy loss spectrum in the presence of an isolated sphere is shown by a dotted curve for an electron moving with the same velocity and impact parameter. (b) The same as (a) for $b=55 \mathrm{~nm}$. (c) Distribution of photon emission probability per sphere and energy range over azimuthal angles $\varphi$ under the same conditions as in (b). The contour curves limiting white areas correspond to a value of $6 \times 10^{-4} \%$ per $\mathrm{eV}$ and per degree. Consecutive contour curves are separated by $10^{-4}$ in the same units. The units $\% \mathrm{eV}^{-1}$ refer to one event out of 100 incoming electrons within $1 \mathrm{eV}$ of photon energy range.

$$
\Gamma^{\operatorname{loss}}(\omega)=\frac{1}{\pi \omega} \int d t \operatorname{Re}\left\{e^{-i \omega t} \mathbf{v} \cdot \mathbf{E}^{\text {ind }}\left(\mathbf{r}_{t}, \omega\right)\right\}
$$

can be interpreted as the probability that the electron undergoes a scattering event losing an amount of energy $\omega$. 


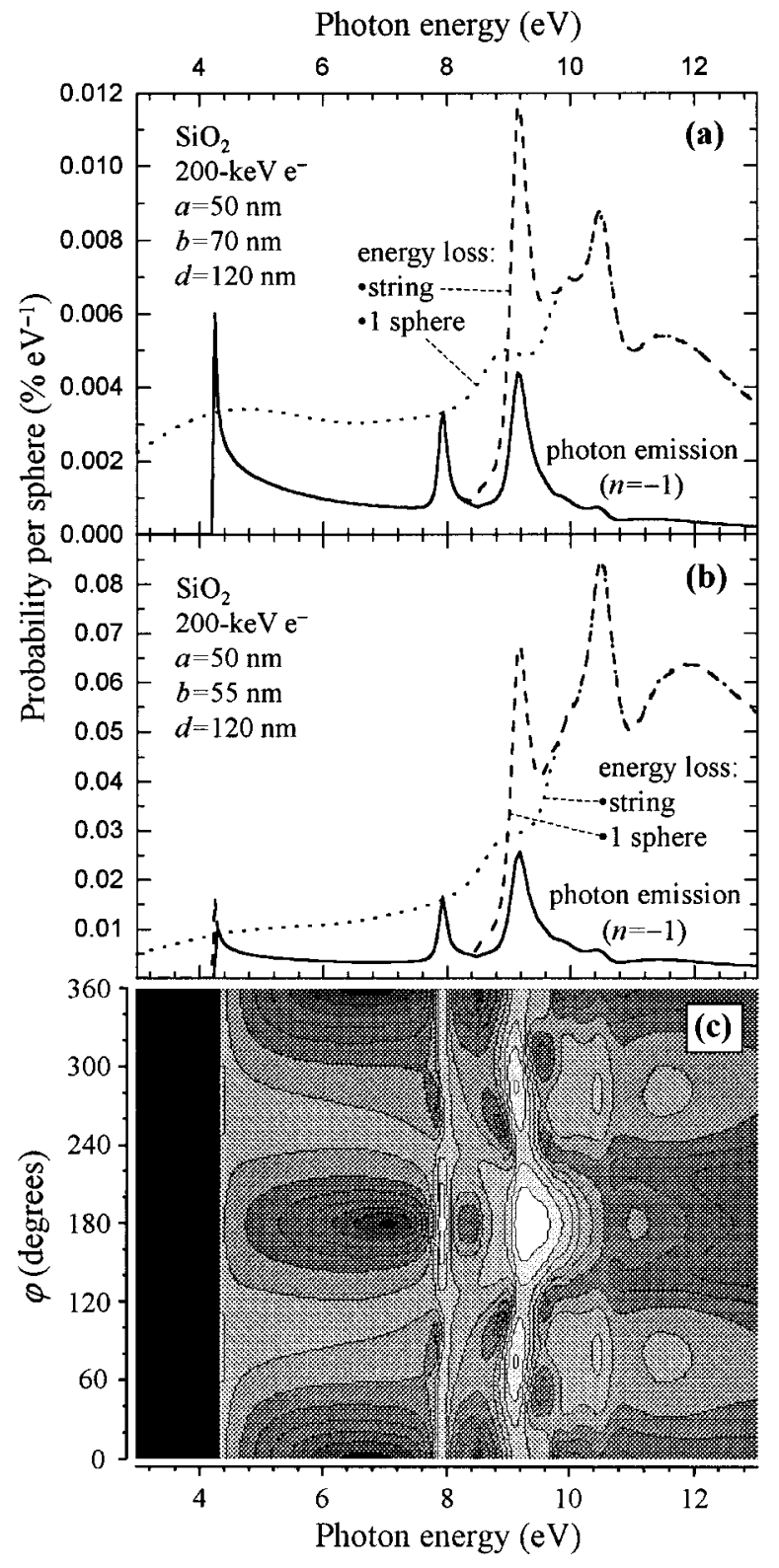

FIG. 3. The same as Fig. 2 for $\mathrm{SiO}_{2}$ spheres. In (c) the contour curves limiting white areas correspond to a value of $10^{-4} \%$ per eV and per degree. Consecutive contour curves are separated by a factor of $10^{0.2}$.

Due to the periodicity of the geometry under consideration (see Fig. 1), the contribution of each object of the string to the loss probability has to be identical. Actually, inserting the multipole expansion of $\psi^{\text {ind }}$ [see Eq. (15)] into Eq. (1), and this in turn into Eq. (27), one finds that the loss probability per sphere reduces to [30]

$$
\begin{aligned}
\frac{\Gamma^{\operatorname{loss}}(\omega)}{N}= & \frac{1}{\pi \omega^{2}} \sum_{L} K_{m}\left[\frac{\omega b}{v \gamma}\right] \operatorname{Re}\left\{m v\left(A_{L}^{+}\right)^{*} i^{l} \phi_{L}^{M, \text { ind }}\right. \\
& \left.+\frac{c}{2 \gamma} B_{L}^{*} i^{l} \phi_{L}^{E \text {,ind }}\right\},
\end{aligned}
$$

where use of Eq. (15) has been made. Results for the energy loss are given in Figs. 2 and 3 and they are discussed in next section.

\section{RESULTS FOR STRINGS OF SPHERES}

The central results of Sec. II are Eqs. (25) and (28). The former represents the probability of photon emission per incident electron and per sphere for the geometry of Fig. 1. The emission is decomposed into different harmonics $n$, each of which contributes with a well defined polar angle $\theta_{n}$ for each photon energy $\omega$, as given by Eq. (26). Equation (28) represents the electron energy loss probability per sphere. These probabilities are shown in Figs. 2-7 and they have been normalized to the number of spheres $N$ and the energy range, so that, for instance, a probability of photon emission (or energy loss) of $0.1 \% \mathrm{eV}^{-1}$ in Fig. 2(b) represents the emission of $0.001 \mathrm{~N}$ photons per incoming electron (or the detection of a fraction of $0.001 \mathrm{~N}$ electrons) within an energy range of $1 \mathrm{eV}$.

Besides the parameters shown in Fig. 1, these expressions require to input the scattering properties of the spheres in the string, that is, their scattering matrices. Clusters formed by homogeneous spheres made of $\mathrm{Al}$ and $\mathrm{SiO}_{2}$ are considered next. Their scattering matrices have been calculated using the analytical expression $[29,30]$

$$
t_{L L^{\prime}}=\delta_{L L^{\prime}}\left[\begin{array}{cc}
t_{l}^{M} & 0 \\
0 & t_{l}^{E}
\end{array}\right],
$$

where

$$
\begin{gathered}
t_{l}^{M}=\frac{-j_{l}\left(\rho_{0}\right) \rho_{1} j_{l}^{\prime}\left(\rho_{1}\right)+\rho_{0} j_{l}^{\prime}\left(\rho_{0}\right) j_{l}\left(\rho_{1}\right)}{h_{l}^{(+)}\left(\rho_{0}\right) \rho_{1} j_{l}^{\prime}\left(\rho_{1}\right)-\rho_{0}\left[h_{l}^{(+)}\left(\rho_{0}\right)\right]^{\prime} j_{l}\left(\rho_{1}\right)}, \\
t_{l}^{E}=\frac{-j_{l}\left(\rho_{0}\right)\left[\rho_{1} j_{l}\left(\rho_{1}\right)\right]^{\prime}+\epsilon\left[\rho_{0} j_{l}\left(\rho_{0}\right)\right]^{\prime} j_{l}\left(\rho_{1}\right)}{h_{l}^{(+)}\left(\rho_{0}\right)\left[\rho_{1} j_{l}\left(\rho_{1}\right)\right]^{\prime}-\epsilon\left[\rho_{0} h_{l}^{(+)}\left(\rho_{0}\right)\right]^{\prime} j_{l}\left(\rho_{1}\right)},
\end{gathered}
$$

$\rho_{0}=k a, \rho_{1}=k a \sqrt{\epsilon}$ (here, $\operatorname{Im}\left\{\rho_{1}\right\}>0$ ), $a$ is the sphere radius, $\epsilon$ is the frequency-dependent dielectric function of $\mathrm{Al}$ and $\mathrm{SiO}_{2}$, respectively, and the prime denotes differentiation with respect to $\rho_{0}$ and $\rho_{1}$. In particular, the response of $\mathrm{Al}$ has been approximated by a Drude dielectric function with bulk plasma energy $\omega_{p}=15 \mathrm{eV}$ and damping $\eta=1.06 \mathrm{eV}$. The dielectric function of $\mathrm{SiO}_{2}$ has been taken from optical data [38].

These two different materials have been chosen because they represent prototypical cases of metals and insulators, respectively. Since the latter are unable to attenuate radiation within their energy gaps, one would expect larger photon emission probabilities in this case. However, although part of the energy lost by the electron is dissipated in the medium in the case of metals, larger intensities are found in this case, as shown below.

The radiation emission probabilities have been calculated from Eqs. (21) and Eq. (25), where the coefficients $\phi_{L}^{\text {ind }}$ are obtained by solving Eq. (13) with the external field given by Eq. (11) as input. The operators appearing in these equations have been approximated by finite matrices of dimension $l_{\max }-|m|+1$ for each azimuthal number $m$, where $l_{\max }$ is the maximum value of the orbital angular momentum number under consideration. Convergence has been achieved for $l_{\max }=8$ in the examples offered below. 


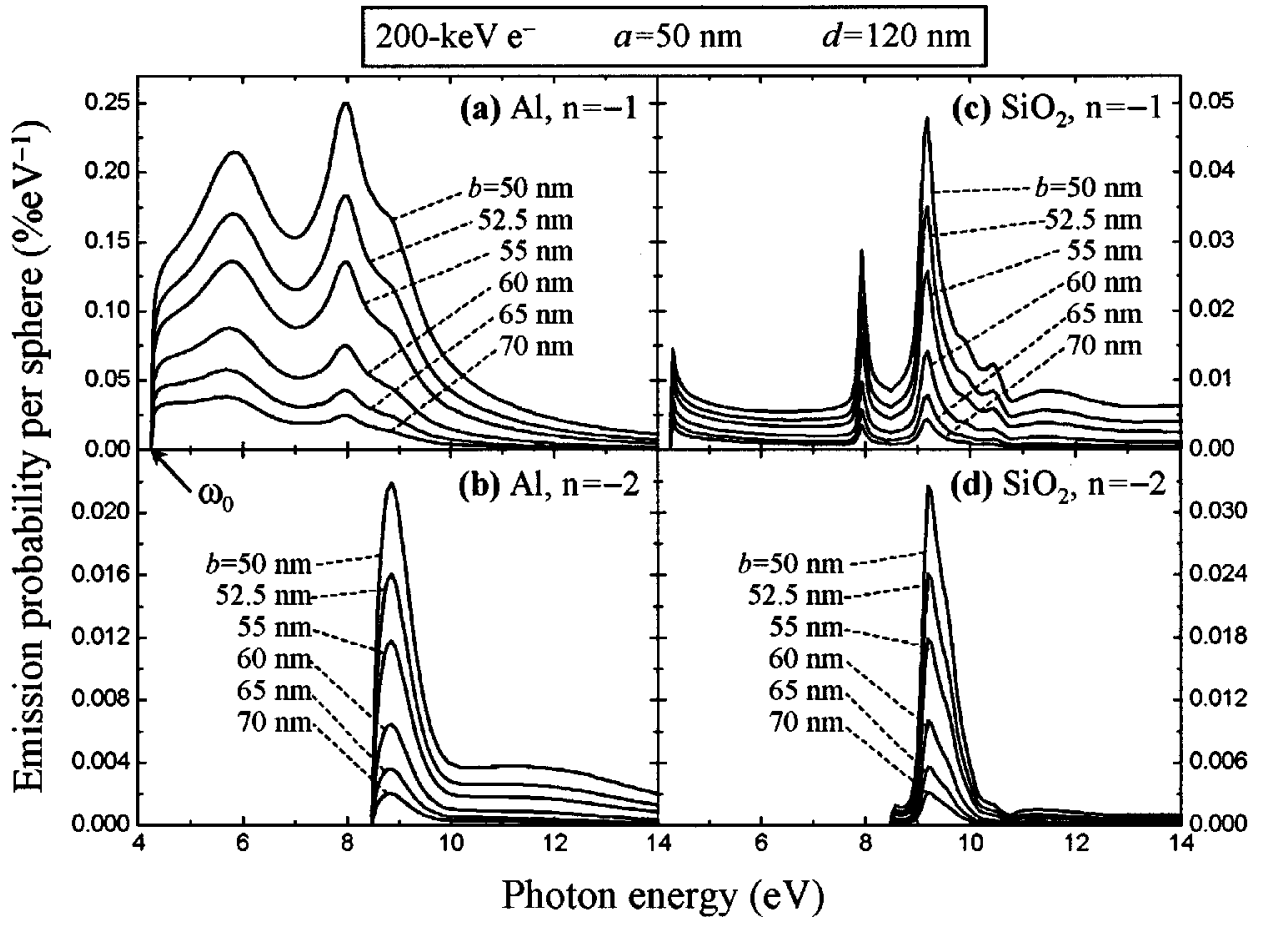

FIG. 4. (a) Probability of photon emission per sphere and energy range for $200-\mathrm{keV}$ electrons moving parallel to a string of $\mathrm{Al}$ spheres, as calculated from Eq. (25) for only $n=-1$ (first harmonic). The string spacing is $d$ $=120 \mathrm{~nm}$ and the sphere radius is $a=50 \mathrm{~nm}$. Different values of the impact parameter $b$ have been considered, as shown in the figure. (b) The same as (a) for $n=-2$. (c) The same as (a) for $\mathrm{SiO}_{2}$ spheres. (d) The same as (a) for $n=-2$ and $\mathrm{SiO}_{2}$ spheres.
Figures 2 and 3 illustrate the energy loss and induced photon emission probabilities for $200-\mathrm{keV}$ electrons moving parallel to an infinite periodic string of aligned $\mathrm{Al}$ and $\mathrm{SiO}_{2}$ spheres, respectively, as shown in Fig. 1. In the case of $\mathrm{SiO}_{2}$ (Fig. 3), the energy loss probability per sphere [dashed curves, calculated from Eq. (28)], which is proportional to the electron intensity that could be measured in an EELS experiment, is seen to follow the photon emission probability [solid curves, including only $n=-1$, and calculated by integrating Eq. (25) over azimuthal angles $\varphi$ ] within the region below $8.5 \mathrm{eV}$, where the imaginary part of the dielectric function is very small and most of the energy loss is converted into SP radiation, leading to an absolute threshold for energy losses at $\omega_{0} \approx 4.24 \mathrm{eV}$ under the conditions of the figure. In $\mathrm{Al}$ (Fig. 2), part of the energy loss goes always into absorption. In particular, this is the case in the energy loss peak at $3.8 \mathrm{eV}$ (below the emission threshold), which can be ascribed to the excitation of an intrinsic mode of the string. However, the radiation emission probability is higher in the Al case for $n=-1$ as compared to silica. The energy loss probability (EELS spectrum) for an isolated sphere (dotted curves) can be obtained from Eq. (28) in the $d \rightarrow \infty$ limit, leading to analytical expressions derived elsewhere [30]. The latter follows the loss probability in the string at high energy losses, where the coupling between spheres plays a minor role. However, the low-energy part of the EELS spectrum of an isolated sphere looks very different from that of the string, and in particular, the former takes non-negligible values below the threshold of the latter in the case of silica (see Fig. $3)$.

The distribution of the emission in azimuthal angle $\varphi$ has been represented in Figs. 2(c) and 3(c) for strings of $\mathrm{Al}$ and $\mathrm{SiO}_{2}$ spheres, respectively. These materials exhibit different $\varphi$ dependence, but in both cases the emission is focused on the side of the string opposite with respect to the trajectory.

Figure 4 shows the probability of photon emission per sphere as a function of emission energy for $200-\mathrm{keV}$ electrons moving with different impact parameters $b$ with respect to the axis of an infinite string of aligned spheres made of $\mathrm{Al}$ [Figs. 4(a) and (b)] and $\mathrm{SiO}_{2}$ [Figs. 4(c) and (d)] for $n=$ -1 [Figs. 4(a) and (c)] and $n=-2$ [Figs. 4(b) and (d)]. Notice that the emission is weaker in $\mathrm{SiO}_{2}$ as compared with Al for $n=-1$, in agreement with the results of Figs. 2 and 3. However, the $n=-2$ harmonic has comparable magnitude in both cases, since its threshold occurs at larger energies, where the dielectric function of silica has already a sizable imaginary part. The emission probability increases monotonically with decreasing impact parameter as expected.

The dependence of the angle of emission on photon energy has been represented in Fig. 5(a) for 200-keV electrons and different values of the string spacing $d$, as calculated from Eq. (26) for $n=-1$. The first-harmonic emission takes place within a finite range of energies, as can be seen in Fig. $5(\mathrm{~b})$, where the emission probability per sphere has been plotted for different values of $d$ as a function of photon energy for the case of Al. The bulk of the emission is contained in the region 3-9 eV in all cases. Notice that the position of the maxima of emission at around $6 \mathrm{eV}$ and $8 \mathrm{eV}$ is rather insensitive to the string spacing. Actually, features at these energies are also observed in the loss spectra of isolated Al spheres of the same radius, as shown in Fig. 2, and they can be ascribed to the excitation of dipole and quadrupole modes, respectively, whose energies depend on the sphere radius [30].

Figure 6 shows the dependence of first-harmonic emission spectra on electron energy for two different values of the string spacing, also for Al spheres. Again, the limits imposed by Eq. (26) are translated into a finite energy domain where the emission is allowed. The largest intensities are obtained for electron energies of $200-600 \mathrm{keV}$ in both cases, though the results for the larger separation [Fig. 6(b)] show an overall enhancement of the emission. 


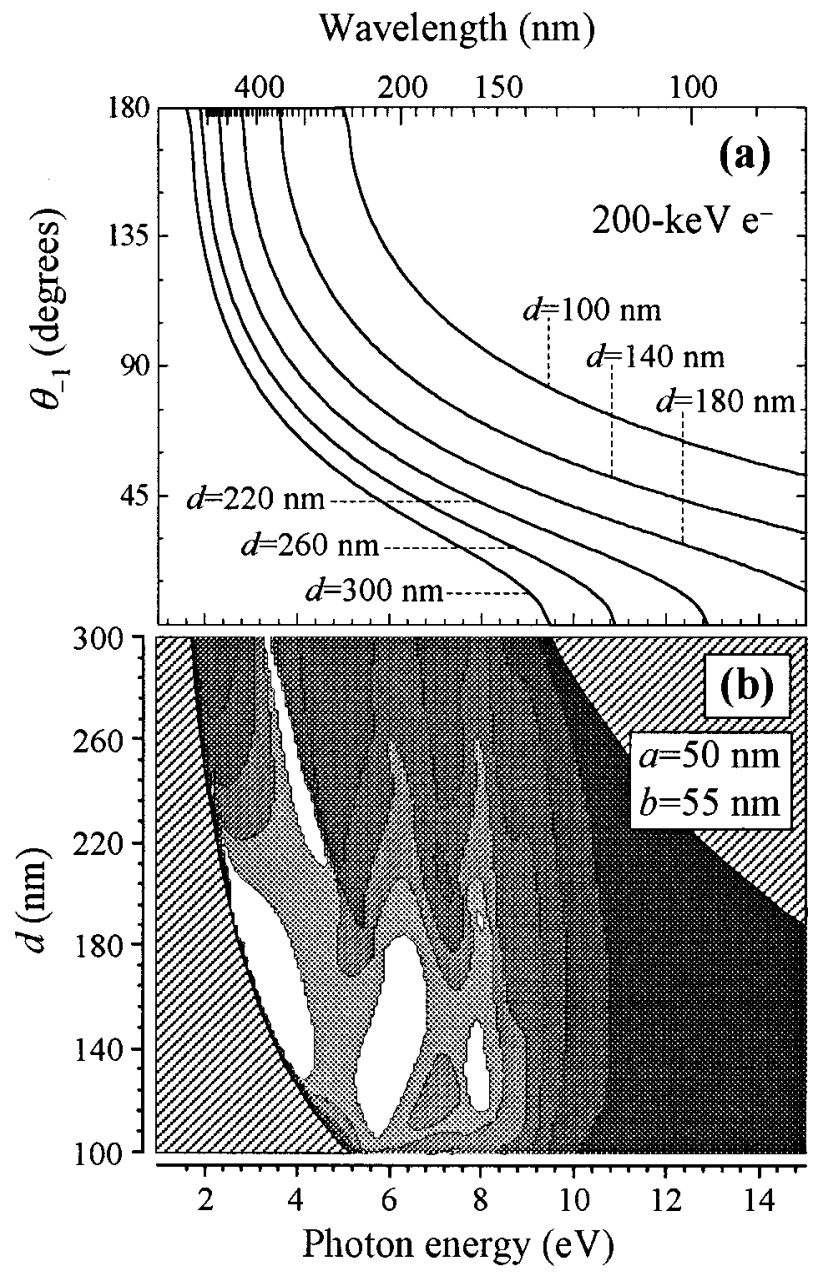

FIG. 5. (a) Dependence of the polar angle of SP emission on photon energy, as calculated from Eq. (26) for $n=-1$ and various values of $d$ (see Fig. 1). (b) Probability of photon emission per sphere and energy range for $n=-1$ as a function of photon energy and spacing $d$ for a string of $\mathrm{Al}$ spheres of radius $a=50 \mathrm{~nm}$. The electron impact parameter is $b=55 \mathrm{~nm}$. The regions where emission is forbidden have been represented by shaded areas. The contour curves limiting white areas correspond to a value of $0.12 \% \mathrm{eV}^{-1}$. Consecutive contour curves are separated by a difference of $0.02 \% \mathrm{eV}^{-1}$.

The effects of termination in the string have been investigated using the method summarized in Sec. II A for solving Maxwell's equations in the presence of a cluster of arbitrarily-distributed objects $[28,31]$. Figure 7 (a) shows the probability of photon emission as a function of photon energy for finite strings of $N$ aligned $\mathrm{Al}$ spheres. Different values of $N$ have been considered, as shown by labels. The distribution of the emission in polar angle $\theta$ has been represented in Figs. 7(b) -7 (f). The larger $N$, the more focused the emission. Actually, upon inspection of Eq. (24), the spread in phase difference in the emission is seen to be proportional to $\delta \Delta \approx 2 \pi / N$, which results in a polar-angle spread given by $\delta \theta \approx 2 \pi c /(N \omega d \sin \theta)$. Under the conditions of Fig. 7 and for $\omega=6 \mathrm{eV}$, this leads to $\delta \theta \approx 50^{\circ} / N$, in good agreement with the results shown in the figure. The increase of polarangle spread with decreasing $\omega$ predicted by the above expression is also observed in the figures.

Notice that two intense regions are shaping up as $N$ in-

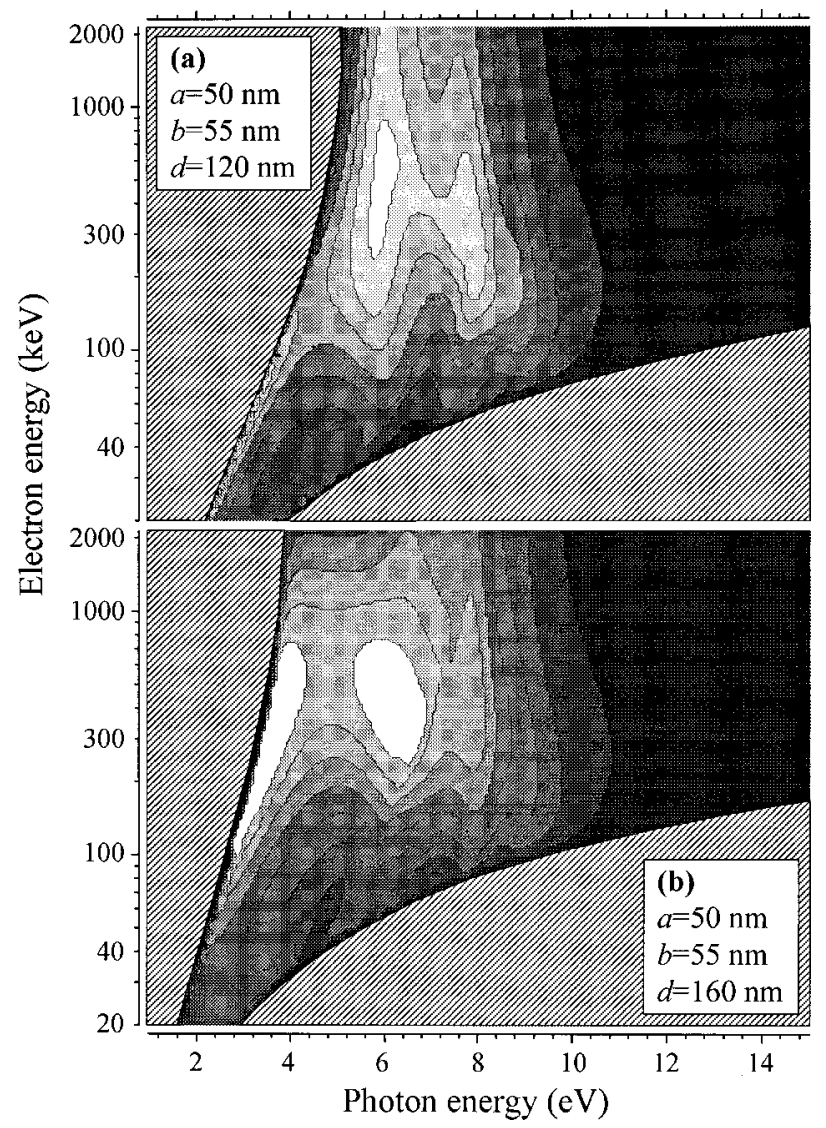

FIG. 6. SP radiation emission probability per sphere and energy range as a function of photon energy and electron energy for the geometry illustrated in Fig. 1 . The sphere radius is $a=50 \mathrm{~nm}$, the impact parameter is $b=55 \mathrm{~nm}$, and the string spacing is $d$ $=120 \mathrm{~nm}$ and $160 \mathrm{~nm}$ in (a) and (b), respectively. The regions where emission is forbidden have been represented by shaded areas. The contour curves limiting white areas correspond to a value of $0.14 \% \mathrm{eV}^{-1}$ and $0.16 \% \mathrm{eV}^{-1}$ in (a) and (b), respectively. Consecutive contour curves are separated by a difference of $0.02 \% \mathrm{eV}^{-1}$ in both cases.

creases in Figs. 7(d) -7 (f). They correspond to $n=-1$ and $n=-2$, respectively, and their profiles converge to the ones given in Fig. 5(a) as $N \rightarrow \infty$.

\section{CONCLUDING REMARKS}

The emission of photons by interaction of fast electrons with aligned strings of nanoparticles has been analyzed theoretically. This constitutes a generalization of the SmithPurcell effect [1], which takes place when an electron moves near a grating. The Maxwell equations have been solved analytically in the presence of a string of homogeneous spheres, and the cases of $\mathrm{Al}$ and $\mathrm{SiO}_{2}$ have been considered for various values of the different parameters involved in the geometry (i.e., electron energy and impact parameter, string spacing, and sphere radius). This approach can be employed to investigate strings formed by arbitrary objects, provided one knows their scattering matrices, and a trivial extension would lead to the simulation of periodic strings with a basis formed by more than just one object.

Under the conditions of Figs. 6(b), where Al spheres of diameter $100 \mathrm{~nm}$ separated $60 \mathrm{~nm}$ are considered, the maxi- 


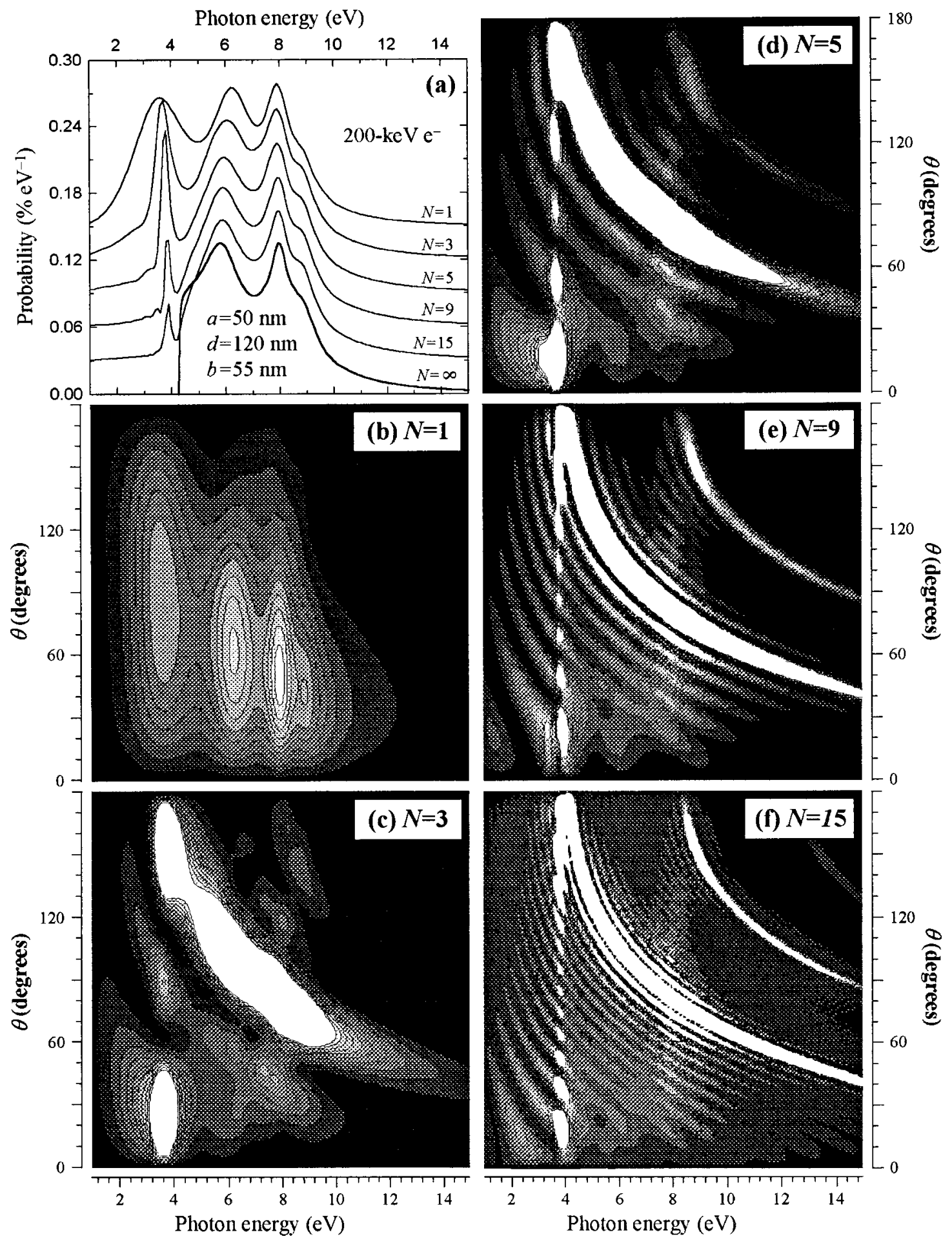

FIG. 7. Probability of photon emission for an electron moving parallel to a finite string of $N$ aligned Al spheres as a function of photon energy and polar angle $\theta$. The emission has been integrated over the azimuthal angle $\varphi$. The geometry is the same as in Fig. 1, except that the string is finite. The values of $N$ under consideration are 1, 3, 9, 15, and 25 in (b)-(f), respectively. The integral over all directions of emission is shown in (a) for different values of $N$, including $N=\infty$. Here, the probability has been normalized to the number of spheres $N$ in each case and consecutive curves have been shifted $0.03 \% \mathrm{eV}^{-1}$ upwards to improve readability.

mum of photon emission occurs when the electron energy is $300 \mathrm{keV}$. For a sphere-electron separation of $5 \mathrm{~nm}$, assumingcoherent emission from $100 \mathrm{Al}$ spheres and an electron in-tensity of $1 \mu \mathrm{A}$ (this could be achieved by using a planar electron beam in interaction with different regions of a plane covered by strings of aligned spheres), the average outgoing flux in the energy region $\omega=4-10 \mathrm{eV}$ is $\sim 10^{12}$ photons per second per $\mathrm{eV}$. This has to be compared with values of $\sim 10^{13}$ on the sample within end stations of third generation synchrotron lines in the same energy range.

Using a more general method to solve exactly the Maxwell equations in the presence of arbitrarily distributed dielectric objects [28,31], finite strings of $\mathrm{Al}$ spheres have been investigated. Strings formed by 50 spheres have been shown 
to be able to narrow the bulk of the emission to within a polar angle window of $\delta \theta=1^{\circ}$.

\section{ACKNOWLEDGMENTS}

The author wants to thank M. A. Van Hove and C. S. Fadley for their kind hospitality during his stay in Berkeley,
A. Howie and Z. Hussain for helpful and enjoyable discussions, and R. Díez for a critical reading of the manuscript. Help and support from the University of the Basque Country and the Spanish Ministerio de Educación y Cultura (Fulbright grant FU-98-22726216) is gratefully acknowledged. Part of this work was supported by the U.S. Department of Energy under Contract No. DE-AC03-76SF00098.
[1] S.J. Smith and E.M. Purcell, Phys. Rev. 92, 1069 (1953).

[2] W.W. Salisbury, J. Opt. Soc. Am. 60, 1279 (1970).

[3] J.P. Bachheimer, Phys. Rev. B 6, 2985 (1972).

[4] P.M. van den Berg, J. Opt. Soc. Am. 63, 1588 (1973).

[5] P.M. van den Berg and T.H. Tan, J. Opt. Soc. Am. 64, 325 (1974).

[6] K. Mizuno, S. Ono, and O. Shimoe, Nature (London) 253, 184 (1975).

[7] J.C. McDaniel, D.B. Chang, J.E. Drummond, and W.W. Salisbury, Appl. Opt. 28, 4924 (1989).

[8] G. Doucas, J.H. Mulvey, M. Omori, J. Walsh, and M.F. Kimmitt, Phys. Rev. Lett. 69, 1761 (1992).

[9] M.J. Moran, Phys. Rev. Lett. 69, 2523 (1992).

[10] O. Haeberlé, P. Rullhusen, J.-M. Salomé, and N. Maene, Phys. Rev. E 49, 3340 (1994); 55, 4675 (1997); P. Henri, O. Haeberlé, P. Rullhusen, N. Maene, and W. Mondelaers, ibid. 60, 6214 (1999).

[11] K.J. Woods, J.E. Walsh, R.E. Stoner, H.G. Kirk, and R.C. Fernow, Phys. Rev. Lett. 74, 3808 (1995).

[12] J.H. Brownell, J. Walsh, and G. Doucas, Phys. Rev. E 57, 1075 (1998).

[13] Y. Shibata, S. Hasebe, K. Ishi, S. Ono, M. Ikezawa, T. Nakazato, M. Oyamada, S. Urasawa, T. Takahashi, T. Matsuyama, K. Kobayashi, and Y. Fujita, Phys. Rev. E 57, 1061 (1998).

[14] J. Urata, M. Goldstein, M.F. Kimmit, A. Naumov, C. Platt, and J.E. Walsh, Phys. Rev. Lett. 80, 516 (1998).

[15] R. Temkin, Science 280, 854 (1998).

[16] A.P. Potylitsyn, Nucl. Instrum. Methods Phys. Res. B 145, 60 (1998).

[17] G. Toraldo di Francia, Nuovo Cimento 16, 61 (1960).

[18] N. Zabala and P.M. Echenique, Ultramicroscopy 32, 327 (1990).
[19] R.H. Ritchie, Phys. Rev. 106, 874 (1957).

[20] R. García-Molina, A. Gras-Martí, A. Howie, and R.H. Ritchie, J. Phys. C 18, 5335 (1985).

[21] T.L. Ferrell and P.M. Echenique, Phys. Rev. Lett. 55, 1526 (1985).

[22] A. Rivacoba, N. Zabala, and P.M. Echenique, Phys. Rev. Lett. 69, 3362 (1992).

[23] F.J. García de Abajo and A. Howie, Phys. Rev. Lett. 80, 5180 (1998).

[24] J.B. Pendry and A. MacKinnon, Phys. Rev. Lett. 69, 2772 (1992).

[25] J.B. Pendry and L. Martín-Moreno, Phys. Rev. B 50, 5062 (1994).

[26] J. Aizpurua, A. Rivacoba, N. Zabala, and F.J. García de Abajo, Surf. Sci. 402-404, 418 (1998).

[27] G. Biasiol and E. Kapon, Phys. Rev. Lett. 81, 2962 (1998).

[28] F.J. García de Abajo, Phys. Rev. Lett. 82, 2776 (1999).

[29] K. Ohtaka and M. Inoue, Phys. Rev. B 25, 677 (1982).

[30] F.J. García de Abajo, Phys. Rev. B 59, 3095 (1999).

[31] F.J. García de Abajo, Phys. Rev. B 60, 6086 (1999).

[32] F.E. Low, Classical Field Theory: Electromagnetism and Gravitation (Wiley, New York, 1997).

[33] A. Messiah, Quantum Mechanics (North-Holland, New York, 1966).

[34] J.B. Pendry, Low Energy Electron Diffraction (Academic Press, London, 1974).

[35] A. Winther and K. Alder, Nucl. Phys. A 319, 518 (1979).

[36] M. Abramowitz and I.A. Stegun, Handbook of Mathematical Functions (Dover Publications, New York, 1972).

[37] J.D. Jackson, Classical Electrodynamics (Wiley, New York, 1975).

[38] E.D. Palik, Handbook of Optical Constants of Solids (Academic Press, New York, 1985). 